\title{
Heavy Metals Effect on Cyanobacteria Synechocystis aquatilis Study Using Absorption, Fluorescence, Flow Cytometry, and Photothermal Measurements
}

\author{
A. Dudkowiak · B. Olejarz • J. Lukasiewicz • \\ J. Banaszek • J. Sikora · K. Wiktorowicz
}

Received: 2 April 2010 / Accepted: 8 October 2010 / Published online: 26 October 2010

(C) The Author(s) 2010. This article is published with open access at Springerlink.com

\begin{abstract}
The toxic effect of six heavy metals on cyanobacteria Synechocystis aquatilis was studied by absorption, fluorescence, flow cytometry, and photothermal measurements. This study indicates that at the concentration used, the cyanobacteria are more sensitive to silver, copper, and mercury than to cadmium, lead, and zinc metals. Disregarding the decrease in the yields of the related radiative processes caused by photochemical processes and/or damage to phycobilisomes, no changes were detected in the efficiency of thermal deactivation processes within a few microseconds, which can indicate the lack of disturbances in the photosynthetic light reaction and the lack of damage to the photosystem caused by the heavy metal ions in the concentrations used. The results demonstrate that the relative values of fluorescence yield as well as promptly generated heat calculated for the metal-affected and unaffected (reference) bacteria are sensitive indicators of environmental pollution with heavy metal ions, whereas the complementary methods proposed could be used as a noninvasive and fast procedure for in vivo assessment of their toxicity.
\end{abstract}

Keywords Chlorophyll - Cyanobacteria - Heavy metal ·

Optical and photothermal spectroscopy $\cdot$ Synechocystis aquatilis

\footnotetext{
A. Dudkowiak $(\varangle) \cdot$ B. Olejarz $\cdot$ J. Łukasiewicz $\cdot$ J. Banaszek

Faculty of Technical Physics, Poznan University of Technology, Nieszawska 13a, 60-965 Poznan, Poland

e-mail: alina.dudkowiak@put.poznan.pl

J. Sikora $\cdot$ K. Wiktorowicz

Department of Biology and Environmental Sciences, K. Marcinkowski University of Medical Sciences,

Długa 1/2, 61-848 Poznan, Poland
} 


\section{Introduction}

Heavy metals in an aqueous environment are hazardous for biological life in water reservoirs and for man using these resources. Elevated levels of heavy metals in the environment have been connected with agricultural and industrial activities [1]. The scale of the problem has prompted the search for effective and reliable ways of monitoring the level of pollution. A very promising method proposed for this purpose is biomonitoring based on observation of living organisms exposed to the effect of hazardous environmental agents and analysis of changes caused by these agents in tissues and cells.

Biomonitoring is a scientific technique for assessing living organism exposures to natural and synthetic chemicals, based on sampling and the quantitative or semiquantitative analysis of biological effects observed in the micro-organisms studied (bioindicators). In contrast to chemical analytical methods, the bioanalytical ones permit direct evaluation of the toxic effect of pollutants on organisms [1,2]. Biomonitoring is performed employing organisms particularly sensitive to a given environmental factor or such that are capable of its effective absorption or accumulation. Aquatic micro-organisms stand in the front line of the pollution exposure. In the presence of excessive amounts of heavy metals, the toxicity symptoms appear as a result of interactions at the cellular/molecular level $[3,4]$. Toxicity can result from the binding of metals or from the displacing of an essential element as well as from the stimulating formation of free radicals and reactive oxygen species. Plants possess some mechanism in heavy metal detoxification and tolerance [4].

The heavy metals are those with a density higher than $5000 \mathrm{~kg} \cdot \mathrm{m}^{-3}$. This group includes 53 naturally existing chemical elements (among them As, which is a semimetal). Many heavy metals play essential roles for living photosynthetic organisms, and a very low concentration of their ions is required for optimum growth in distinct culture media [5]. Most of the chemical elements often occur as cations able to form complex molecules and show activity in redox reactions. In trace amounts heavy metal cations often play important roles in the organisms as they take part in complex biochemical reactions. In higher concentrations they are toxic as they form nonspecific complexes in cells [6]. Among the heavy metals, $\mathrm{Fe}, \mathrm{Cu}, \mathrm{Mn}, \mathrm{Co}, \mathrm{Zn}$, and $\mathrm{Ni}$ are required for correct functioning of living organisms, but an excess or deficit of these metals can promote severe toxic effects, which depend on the type of organism, the nature and concentration of the metal, and environmental conditions accompanying heavy metal stress. However, there are the heavy metals that can be toxic even in small amounts and are not used in cell metabolism such as $\mathrm{Cd}, \mathrm{Pb}$, and $\mathrm{Hg}$. Estimates of water ecosystem pollution with heavy metals by means of bioindicating methods are of special interest [7]. By biological indicators we understand plankton organisms, insects, mollusks, fish, plants, and birds [1]. Cyanobacteria (formerly known as blue-green algae) belong to the group of plankton organisms showing sensitivity to increased concentration of some heavy metals [8-11]. Cyanobacteria are thought to be among the evolutionarily oldest organisms; they can perform oxygenic photosynthesis and respiration simultaneously, and many of the species are able to fix nitrogen [12]. Synechocystis are unicellular, photoautotrophic, facultative glucose-heterotrophic cyanobacteria. They can survive and prosper under a wide range 
of environmental conditions. Despite their high degree of stress tolerance, it has been shown that cyanobacteria are highly sensitive to heavy metal pollution [13,14]; therefore, they can serve as an indicator of the level of metal pollution. The mechanism of influence of heavy metals on the cells of cyanobacteria has not been well explored yet.

One of the possible ways to analyze the harmful influence of chemical factors on the biological functions of algae cells is an investigation of changes in their autofluorescence. Changes in the fluorescence intensity of some photosynthetic pigments (i.e., Chl-like pigments) are recognized as one of the earliest indicators of the physiological stress in photosynthetic organisms $[15,16]$. In many publications $[10,17-19]$, the effect of the influence of heavy metals on the autofluorescence of algae, including cyanobacteria, was presented.

Cyanobacteria have a supra-molecular structure [photosystem II (PS II)] in the thylakoid membrane. The reaction center (RC) complex for initial charge separation is in the center of PS II surrounded laterally by peripheral light-harvesting antenna pigment-protein complexes [20]. They are located within the same plane of the thylakoid membrane. Phycobilisome (PBS), another pigment-protein complex, is attached to PS II [21]. It is known that light energy captured by phycobilisome was transferred to light-harvesting protein, and finally to an RC complex.

The fluorescence emission of photosynthetically active organisms originates mainly from chlorophyll a (Chl a) molecules of PS II [17]. According to the model proposed in the literature [22], during photosynthesis the photochemical reactions in PS II compete with those of fluorescence emission and energy dissipation into heat. Therefore, we decided to use complementary spectroscopic methods, including photothermal spectroscopy, to monitor heavy metal ions toxicity and their influence on the efficiency of radiation and thermal deactivation processes in cyanobacteria.

Absorption, fluorescence, flow cytometry, and photothermal measurements were used to study the toxic effect of metal ions on cyanobacteria Synechocystis aquatilis. The cyanobacteria were cultured in aqueous medium and exposed for $24 \mathrm{~h}$ to heavy metal salts in various concentrations. The study was also undertaken to check if the spectroscopic methods applied will permit the use of cyanobacteria as bioindicators of the degree of pollution with heavy metals in natural water reservoirs.

\section{Measurements}

The cyanobacteria S. aquatilis SAUV.f. Salna (VAARA 1978/CD-3) originating from the Gulf of Finland were obtained from the Institute of Botany in Třeboň (Czech Republic). The cyanobacteria culture was grown in sterile conditions on the nutrient medium (BG-11), at room temperature $\left(25^{\circ} \mathrm{C}\right)$ under constant irradiation with white light $\left(10 \mathrm{~W} \cdot \mathrm{m}^{-2}\right)$ [23]. Water solutions of heavy metal salts $\left(\mathrm{AgNO}_{3}, \mathrm{HgCl}_{2}, \mathrm{CdCl}_{2}\right.$, $\mathrm{Cu}\left(\mathrm{NO}_{3}\right)_{2}, \mathrm{~Pb}\left(\mathrm{CH}_{3} \mathrm{COO}\right)_{2}$, and $\left.\mathrm{ZnCl}_{2}\right)$ were added into the medium in amounts that ensure final concentrations of $(1,10$, or 100) $\mu \mathrm{M}$. The measurements were carried out after $24 \mathrm{~h}$ of incubation.

The absorbance and fluorescence spectra were measured on a UV/vis spectrophotometer (Cary 4000, Varian Inc., Palo Alto, CA, USA) and spectrofluorimeter (F4500, Hitachi, Tokyo, Japan). The fluorescence intensity of cyanobacteria cells gated on the 
basis of laser light scattering was recorded by a flow cytometer (FACScan, BD Biosciences, San Jose, CA, USA) working with an argon laser (15 mW, $488 \mathrm{~nm}$ exciting wavelength). The fluorescence was collected by detection channel recording the emission of wavelengths above $650 \mathrm{~nm}$. Analysis of the cytometric data was carried out with (BD Biosciences, San Jose, CA, USA) software. The heat generated as a result of conversion of light energy absorbed by the pigments of cyanobacteria was measured by the photothermal technique. To record the photothermal signals, the laser-induced optoacoustic spectroscopic (LIOAS) method [24-27] was applied. The LIOAS setup (described in details in [28]) used the nitrogen-dye laser (GL-3300/GL301, Photon Technology Int., Birmingham, NJ, USA) as the excitation source. The sample is placed in a $1 \mathrm{~cm}$ cuvette, and a piezoelectric transducer (V1030, Panametrics Inc., Waltham, MA, USA) serving as a detection system is attached to this cuvette wall. The LIOAS signal amplitude (mV) was recorded by an oscilloscope (OS-3060, GoldStar, Seoul, Korea), and the laser pulse energy ( $E_{\text {las }}$ in $\mathrm{mJ}$ ) was measured by an energy meter (Rj-7620, Laser Probe, Utica, NY, USA). The experimentally obtained maximum LIOAS signal amplitude $\left(\mathrm{H}_{\max }\right)$ is related to the energy of the laser pulse $\left(E_{\text {las }}\right)$ and the absorbance $(A)$ of the sample according to [27]

$$
H_{\text {max }}=K \alpha E_{\text {las }}\left(1-10^{-A}\right),
$$

where $K$ is a constant depending on the geometry of the experimental setup and the thermoelastic properties of the solvent, $\alpha$ is the fraction of the energy absorbed and released as heat within the time resolution of the setup, in the range of ( 0 to 0.5$) \mu$ s. The factor $\left(1-10^{-\mathrm{A}}\right)$ gives the fraction of the incident light energy absorbed by the sample. The fluorescence emission and photothermal signal obtained from a series of both metal-affected and unaffected (reference) cyanobacteria were measured under exactly the same conditions. The results presented are representative of three independent series of measurements and analyses.

\section{Results}

Exemplary absorption spectra of $S$. aquatilis obtained at various metal salt concentrations are presented in Fig. 1. The spectra reflect the absorption of light by pigments, their various species, and pigment-protein complexes present in cyanobacteria. As shown in Fig. 1, the broad absorption band with a maximum at $440 \mathrm{~nm}$ and with a shoulder at about $480 \mathrm{~nm}$ could be assigned to the Soret band of Chl $a$ and carotenoids, whereas the bands at $625 \mathrm{~nm}$ and $678 \mathrm{~nm}$ are related to phycobilisomes (built of phycobilins, e.g., phycoerythrin (PE), phycocyanin (PC), and allophycocyanin (APC)) and Q-band of Chl $a$. It is known that the size and composition of phycobilisomes can change depending on the culture conditions [20,21], but PE exhibits usually the main absorption maximum at $500 \mathrm{~nm}$ to $565 \mathrm{~nm}$ with the emission one at $585 \mathrm{~nm}$ to $620 \mathrm{~nm}, \mathrm{PC}$ - at $620 \mathrm{~nm}$ to $630 \mathrm{~nm}$ and at $640 \mathrm{~nm}$ to $650 \mathrm{~nm}$, respectively, and for APC, these maxima are at about $650 \mathrm{~nm}$ and $660 \mathrm{~nm}$ to $665 \mathrm{~nm}$. As shown in Fig. 1a, the major peak at $625 \mathrm{~nm}$ was gradually decreased and almost disappeared in the presence of $100 \mu \mathrm{M} \mathrm{Cu}^{2+}$, while the presence of $\mathrm{Cd}$ ions did not disturb significantly 

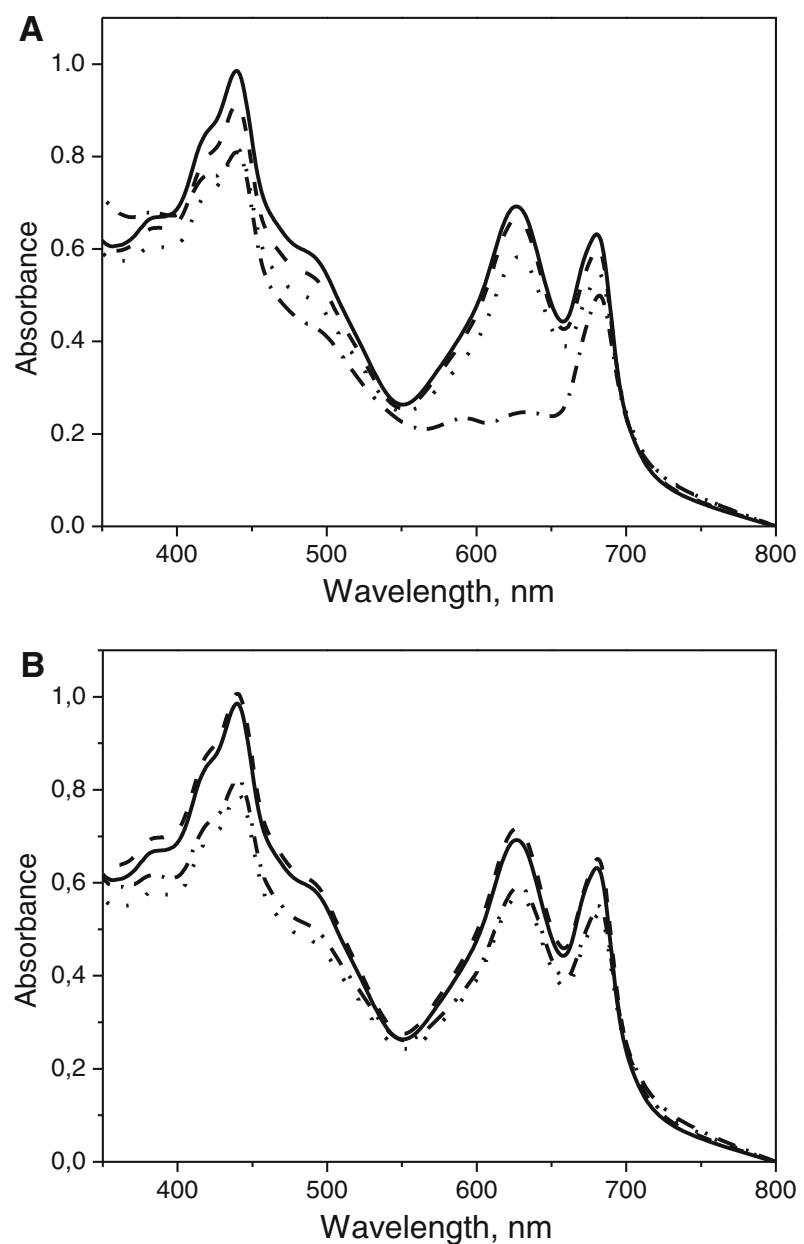

Fig. 1 Influence of (a) $\mathrm{Cu}$ or (b) $\mathrm{Cd}$ on absorption spectra of S. aquatilis cyanobacteria, description of curves: 0 (solid line), $1 \mu \mathrm{M}$ (dashed line), $10 \mu \mathrm{M}$ (dotted line), $100 \mu \mathrm{M}$ (dashed-dotted line)

the absorption spectra of the cyanobacteria tested (Fig. 1b). The results for $\mathrm{Cu}$ ions showed that the amount of phycobilisomes was highly decreased in the culture conditions, and/or the toxicity of the metal ions caused a significant disintegration of this antenna complex. Similarly, a strong influence on absorption as that of $\mathrm{Cu}^{2+}$ ions was observed also for $\mathrm{Ag}^{1+}$ and $\mathrm{Hg}^{2+}$, especially when used in the highest concentrations. On the other hand, the absorption spectra of cyanobacteria treated by $\mathrm{Cd}^{2+}$ are similar to those obtained for cyanobacteria in the presence of the other ions $\left(\mathrm{Zn}^{2+}\right.$ and $\left.\mathrm{Pb}^{2+}\right)$.

These results are strongly correlated with changes in the shape and the intensity of the fluorescence emission (Fig. 2) and excitation spectra (not shown). When the cyanobacteria were excited with blue light $(406 \mathrm{~nm})$ a major emission at $680 \mathrm{~nm}$ with a shoulder at around $650 \mathrm{~nm}$ could be observed. The fluorescence emission is attributed to the Chl $a$ associated with PS II and the phycobilisomes. In the presence of metal 

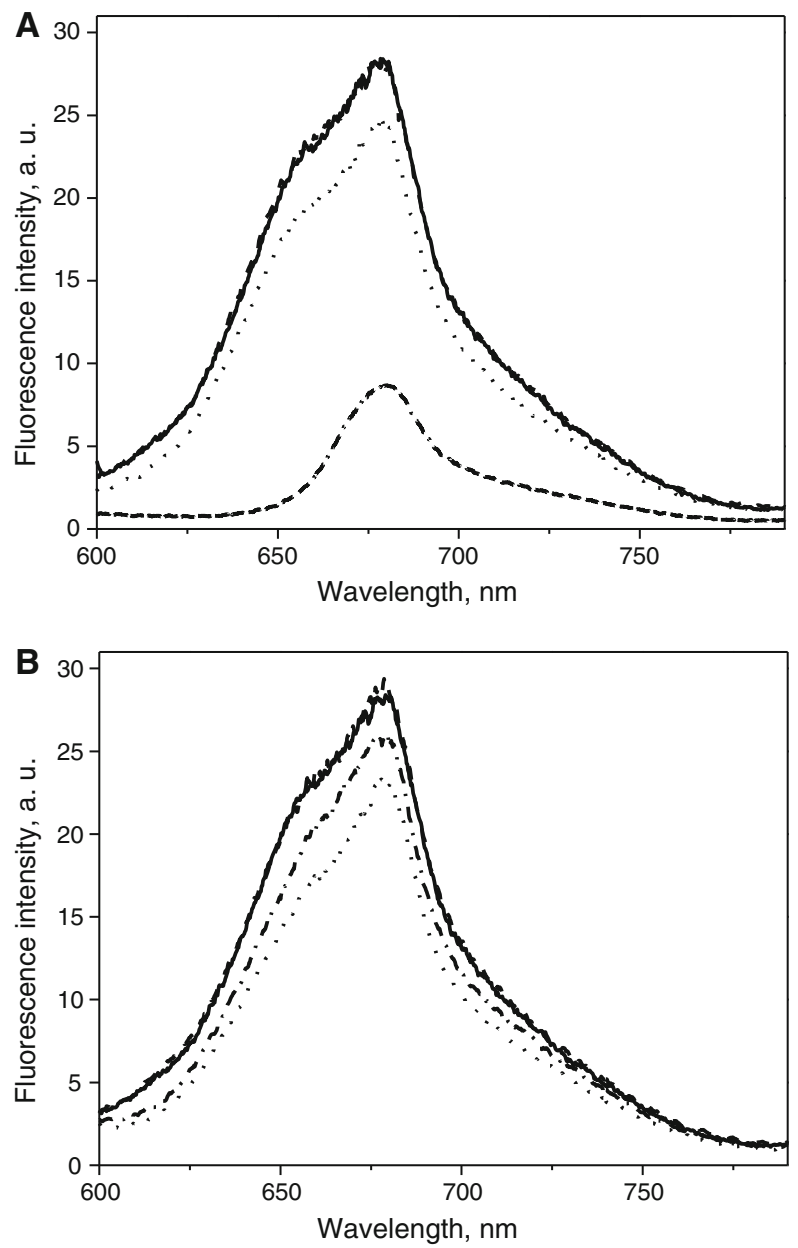

Fig. 2 Changes in the fluorescence emission of cyanobacteria as a result of the presence of (a) $\mathrm{Cu}$ or (b) $\mathrm{Cd}, \lambda_{\text {exc }}=406 \mathrm{~nm}$, description of curves as in Fig. 1

ion concentrations ranging from $1 \mu \mathrm{M}$ to $100 \mu \mathrm{M}$, the fluorescence emission spectra not only altered their strength (Fig. 2 b) but also their shape, e.g., at the highest $\mathrm{Cu}^{2+}$ concentration (Fig. 2a). A shoulder at around $650 \mathrm{~nm}$ almost disappeared probably because the fluorescence from the phycobilisomes did not bring any contribution and the shape of the fluorescence spectrum resembled that of Chl.

Using the excitation wavelength of $488 \mathrm{~nm}$ (the same wavelength was available in the cytometer) that is in the spectral region of carotenoids absorption (not shown), we attempted at comparing the sensitivities of the spectroscopic and cytometric methods and analyzing the type of information that can be obtained from two independent methods based on fluorescence detection. We also wanted to find out if it is possible to get information on the influence of the metal ions on the energy transfer between accessory pigments (carotenoids, phycobilins) and Chl-PS II antenna. The spectral parameters 
Table 1 Ratios of estimated parameters for metal-affected (S) and unaffected (R) Synechocystis aquatilis cyanobacterium

\begin{tabular}{|c|c|c|c|c|c|}
\hline \multirow[t]{2}{*}{ Metal ions } & \multirow[t]{2}{*}{ Conc. $(\mu \mathrm{M})$} & $\mathrm{MFC}_{\mathrm{S}} / \mathrm{MFC}_{\mathrm{R}}( \pm 0.15)$ & $\Phi_{\mathrm{F}}^{\mathrm{S}} / \Phi_{\mathrm{F}}^{\mathrm{R}}( \pm 0.08)$ & $\Phi_{\mathrm{F}}^{\mathrm{S}} / \Phi_{\mathrm{F}}^{\mathrm{R}}( \pm 0.08)$ & $\alpha_{\mathrm{S}} / \alpha_{\mathrm{R}}( \pm 0.05)$ \\
\hline & & \multicolumn{2}{|l|}{$\lambda_{\mathrm{exc}}=488 \mathrm{~nm}$} & \multicolumn{2}{|l|}{$\lambda_{\text {exc }}=406 \mathrm{~nm}$} \\
\hline \multirow[t]{3}{*}{$\mathrm{Ag}^{1+}$} & 1 & 0.97 & 0.98 & 0.97 & 0.92 \\
\hline & 10 & 0.23 & 1.65 & 1.28 & 0.94 \\
\hline & 100 & 0.04 & 0.41 & 0.55 & 0.98 \\
\hline \multirow[t]{3}{*}{$\mathrm{Cd}^{2+}$} & 1 & 1.24 & 0.96 & 1.01 & 0.96 \\
\hline & 10 & 1.52 & 1.13 & 1.02 & 0.96 \\
\hline & 100 & 1.20 & 1.12 & 1.08 & 0.95 \\
\hline \multirow[t]{3}{*}{$\mathrm{Cu}^{2+}$} & 1 & 1.46 & 1.04 & 1.06 & 0.98 \\
\hline & 10 & 0.20 & 1.19 & 1.05 & 0.98 \\
\hline & 100 & 0.03 & 0.38 & 0.37 & 0.99 \\
\hline \multirow[t]{3}{*}{$\mathrm{Hg}^{2+}$} & 1 & 0.54 & 0.99 & 1.11 & 0.93 \\
\hline & 10 & 0.05 & 0.55 & 1.10 & 0.90 \\
\hline & 100 & 0.03 & 0.39 & 0.53 & 0.93 \\
\hline \multirow[t]{3}{*}{$\mathrm{Pb}^{2+}$} & 1 & 0.93 & 1.12 & 1.06 & 0.96 \\
\hline & 10 & 1.26 & 1.10 & 0.93 & 0.94 \\
\hline & 100 & 1.04 & 1.20 & 0.94 & 0.91 \\
\hline \multirow[t]{3}{*}{$\mathrm{Zn}^{2+}$} & 1 & 0.90 & 1.08 & 1.08 & 0.97 \\
\hline & 10 & 1.43 & 1.16 & 1.06 & 0.98 \\
\hline & 100 & 1.39 & 1.21 & 1.18 & 1.01 \\
\hline
\end{tabular}

Numbers given in parentheses near the parameters are the maximum errors

Conc. metal ions concentration, $M F C$ mean fluorescence channel, $\Phi_{\mathrm{F}}$ fluorescence yield, $\alpha$ fraction of the energy absorbed and released as heat within the setup time resolution, $\lambda_{\text {exc }}$ wavelength of excitation

of the absorption, fluorescence emission, and excitation spectra were not significantly different from those of the earlier recorded spectra at the excitation wavelength of $406 \mathrm{~nm}$ (Table 1).

As follows from a comparison of the fluorescence excitation spectra (recorded at $690 \mathrm{~nm}$ ) and the absorption spectra, in the control samples (unaffected cyanobacteria) and in the cyanobacteria in the presence of weakly toxic metal ions, the emission of fluorescence is the responsibility of phycobilisomes and Chl. In the cyanobacteria in the presence of metals considerably influencing the phycobilisomes of cyanobacteria (especially when in concentrations of $100 \mu \mathrm{M}$ ), the emission of fluorescence is related only to Chl $a$ molecules attached to the PS II internal antenna system. This observation suggests the total disintegration of the phycobilisomes and/or disturbances in the process of energy transfer inside and between the antenna systems of photosynthetic apparatus.

On the basis of electronic absorption and fluorescence emission spectra, the ratios of the fluorescence quantum yield $\left(\Phi_{\mathrm{F}}\right)$ for the metal-affected (S) and unaffected (R) cyanobacteria were evaluated (Table 1 ). The relative $\Phi_{\mathrm{F}}$ values were estimated using the following equation:

$$
\frac{\Phi_{\mathrm{F}}^{\mathrm{S}}}{\Phi_{\mathrm{F}}^{\mathrm{R}}}=\frac{I_{\mathrm{S}}}{I_{\mathrm{R}}} \frac{A_{\mathrm{R}}}{A_{\mathrm{S}}},
$$


where $I$ is the area under the fluorescence curve, $A$ is the absorbance at the excitation wavelength, and subscripts $\mathrm{S}$ or $\mathrm{R}$ described the sample and reference, respectively.

The impact of the heavy metal ion concentration on the fluorescence of $S$. aquatilis cells was also measured by the flow cytometry method and estimated by calculating the relative value of the mean fluorescence channel (MFC) for the sample studied and for the reference.

Independent of the detection method used, the relative changes in MFCs and fluorescence yield after $24 \mathrm{~h}$ incubation of cyanobacteria in the presence of the heavy metal ions studied showed similar trends (Table 1). Differences between the quantitative and the qualitative information obtained by stationary fluorescence spectroscopy and flow-through cytometry follow from the method of detection and final presentation of results. From cytometry we get information on the relative ratio of fluorescence emission of cyanobacteria but no information on changes in absorption at the excitation wavelength caused by toxic agents. Fluorescence is measured in the channel cutting off the radiation of wavelengths below $650 \mathrm{~nm}$ (related to phycobilisomes), and the cytometer does not offer a possibility of changing the excitation wavelength into the wavelength optimum for a given system. Combined use of the emission and absorption spectra permits calculation of the relative quantum yield of fluorescence. Analysis of the absorption spectra shows that in the Chl Soret band, in which the excitation of the sample takes place, the intensity of the band changes but its shape is not significantly changed in the presence of metal ions. Absorbance is substituted into Eq. 2, and the form of the equation implies that the areas under the emission curves have great influence on the final values. It is known that the quantum yield of fluorescence of a dye depends on its aggregation type and the interaction with the environment. Therefore, the relative MFC values are sensitive to changes in the emission intensity, while the relative values of $\Phi_{\mathrm{F}}$ of the sample and the reference bring information on the source of the changes and describe then quantitatively. As follows from an analysis of the results (Table 1), for excitation in the Chl Soret band, the relative yield of fluorescence higher than unity can be related to deaggregation of the dyes or their complexes with proteins and/or to enhancement of the pigment's emission as a result of plasmon generation (observed earlier for some concentrations of Ag ions in Synechocystis [29]). The ratio of $\Phi_{\mathrm{F}}$ of the sample studied and the reference lower than unity (Table 1) mean smaller areas under the fluorescence curves and/or absorbance and suggest disturbances in the energy transfer between phycobilisomes and Chl. Finally, it can be concluded that the emission could be quenched as a consequence of interactions with the ions [29] and/or damaged phycobilisomes can lead to disappearance of their fluorescence caused by the response of cyanobacteria to strong toxicity of metal ions in their environment. It seems that cytometry is more sensitive as it shows that even moderate stress (intermediate ion concentrations) disturbing the electron transfer and the photochemical reaction (according to the mechanism proposed [22]) can be manifested by greater loss of the energy absorbed via fluorescence (when heat emission is relatively constant, see Fig. 3, Table 1) and decrease the emission under stronger stress (higher concentration). A similar effect of fluorescence fluctuations as a function of the incubation time was reported for cyanobacteria affected by $\mathrm{Zn}$ ions [5].

The $H_{\max }$ of LIOAS signals (Fig. 3) allow estimates of the ratio of the $\alpha_{\mathrm{S}} / \alpha_{\mathrm{R}}$ coefficients (Table 1) giving the ratio of the fast thermal energy deactivations of the 

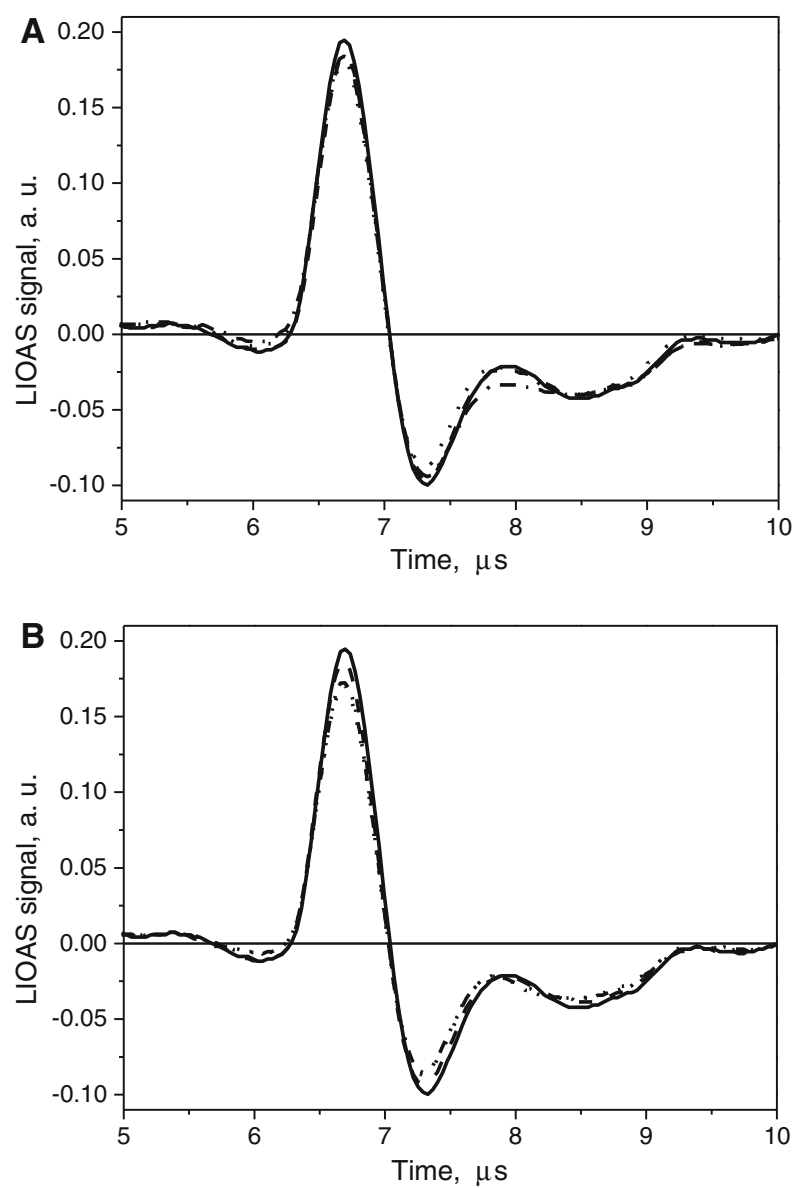

Fig. 3 LIOAS signals of cyanobacteria unaffected and affected by (a) $\mathrm{Cu}$ or (b) $\mathrm{Cd}, \lambda_{\operatorname{exc}}=406 \mathrm{~nm}$, description of curves as in Fig. 1

sample and the reference. Since $A_{\mathrm{S}}$ and $A_{\mathrm{R}}$ can readily be obtained from absorption spectra measured for the sample studied and the reference, the relative value of $\alpha_{S} / \alpha_{R}$ for metal-affected and unaffected bacteria can be calculated using the equation,

$$
\frac{\alpha_{\mathrm{S}}}{\alpha_{\mathrm{R}}}=\frac{H_{\mathrm{max}}^{\mathrm{S}} E_{\text {las }}^{\mathrm{R}}\left(1-10^{-A_{\mathrm{R}}}\right)}{H_{\mathrm{max}}^{\mathrm{R}} E_{\text {las }}^{\mathrm{S}}\left(1-10^{-A_{\mathrm{S}}}\right)} .
$$

Assuming that $\alpha_{\mathrm{R}}$ for native (unaffected) cyanobacteria is used as a reference value, the changes in the amount of energy exchanged into heat as a result of metal ion influence on fast thermal deactivation processes can be estimated. The light reaction of photosynthesis is considered as composed of the photosynthetic primary reactions beginning from the absorption of photons that induces charge separation in a special pair (chlorophyll dimer) and the transfer of electrons in photochemical reactions, and 
the light-dependent reactions such as the electron transport chain and ATP synthesis [30]. As a result of the photosynthetic dark reactions, carbon dioxide is reduced to the carbohydrates, taking place in the time scale from milliseconds to a few minutes. By the laser-induced photothermal technique, it is more suitable to study the photosynthetic light reactions (mainly primary reactions) taking place in a few microseconds after photon absorption than dark reactions needing longer times for completion.

On the basis of the results obtained (Table 1), it was shown that for the concentrations applied, independent of the metal type in the medium environment, the part of energy transformed into heat within the (0 to 0.5$) \mu$ s range is the same (at the assumed level of accuracy) for cyanobacteria in the natural environment and that modified by the presence of metal ions. The effect of lead on the photosynthetic light storage efficiency was measured by the photoacoustic method [31]. It was reported [31] that lead accumulation affects the most initial steps of photochemistry (at concentrations above $120 \mu \mathrm{M}$ ). The advantage of the photoacoustic method is its directness because the signal obtained is proportional to the fraction of light energy that is dissipated as heat. It increases if the photosystem is damaged, and as a consequence, less energy is stored photochemically. According to the results presented, the constant amount of the excitation energy converted into heat suggests that the PS II was not damaged and only its external antennae systems were affected.

Butler [22] has proposed a simple model describing the possible fate of light energy absorbed by Chl associated with PS II. Light energy absorbed by PS II can be used to drive photochemistry [i.e., to transfer an electron from the reaction center Chl (P680) to the primary electron acceptor of PS II $\left.\left(\mathrm{Q}_{\mathrm{A}}\right)\right]$. The processes of photochemistry, Chl fluorescence, and heat loss are in direct competition; therefore, if the rate of one process increases, the rates of the others will decrease [16]. Deactivation of the excitation energy in the form of heat results in nonphotochemical quenching of fluorescence. Our observation allows drawing a conclusion that the decreased fluorescence efficiency of $\mathrm{Chl}$ is rather not connected with nonphotochemical quenching of emission and that the metal ions do not disturb the initial stages of photochemistry.

It was also shown [32] that the stored photosynthetic energy represents the portion of absorbed energy that is not released as heat in photochemically active samples but is stored into chemical intermediates. The stored part of the energy was not affected by the treatment of thylakoids with $\mathrm{Pb}$ ions (a large portion of the energy stored remained unaffected), whereas the maximum decrease in the photosynthetic energy stored was obtained in the presence of $\mathrm{Hg}$ and $\mathrm{Cu}$ ions. Therefore, it seems that $\mathrm{Cu}$ ion toxicity is similar to that of $\mathrm{Hg}$ ions even at low concentrations as follows from our results (Table 1) and the literature [32,33].

The nature of the fluorescence emission intensity changes in the samples reflects the toxic influence of metal ions on S. aquatilis cyanobacteria, and it depends on the type and concentration of the metal ions used. According to the toxicity toward cyanobacteria measured by changes in the autofluorescence of $S$. aquatilis, the heavy metals tested can be divided into two groups. The first one includes $\mathrm{Hg}, \mathrm{Ag}$, and $\mathrm{Cu}$ ions showing strong toxic effects (in the concentration range studied) on cyanobacteria, manifested by changes in the spectral properties observed for $S$. aquatilis. The second group comprises $\mathrm{Cd}, \mathrm{Zn}$, and $\mathrm{Pb}$ ions whose presence does not have a significant effect on the processes of photochemistry and does not disturb the structure of cyanobacteria 
as well as the efficiency of radiative and nonradiative deactivation processes, including the energy transfer between phycobilisomes and chlorophyll. The ordering of metals according to the toxicity toward micro-organisms defined by the minimum inhibitory concentration (MIC) method is as follows: $\mathrm{Hg}>\mathrm{Ag}>\mathrm{Cd}>\mathrm{Cu}>\mathrm{Zn}>\mathrm{Pb}$ [6], which to some extent confirms the results of our measurements. The results also demonstrate that both fluorescence spectroscopy and flow-through cytometry provide data indication of similar trends of the metal ion toxicity regarding the organisms tested.

Using flow-through cytometry and optical spectroscopy and, in particular, the photothermal method, it was shown that the correlation between the excitation energy exchanged into heat and the energy lost as emitted radiation gives information about possible nonphotochemical or photochemical fluorescence quenching. The results demonstrate that the relative ratios of fluorescence as well as the heat generated (in the microsecond time range) calculated for the metal-affected and the reference (unaffected) cyanobacteria are sensitive indicators of metal environment pollution, i.e., permit identification of potent inhibitors of energy transfer and/or electron transport and detect the factors (metal ions) that simultaneously can disturb the primary processes of photosynthesis (the light reaction). It seems that the combination of the methods applied can be used as a noninvasive and fast procedure for in vivo assessment of heavy metal toxicity.

Acknowledgment This study was supported by Poznan University of Technology.

Open Access This article is distributed under the terms of the Creative Commons Attribution Noncommercial License which permits any noncommercial use, distribution, and reproduction in any medium, provided the original author(s) and source are credited.

\section{References}

1. Q. Zhou, J. Zhang, J. Fu, J. Shi, G. Jiang, Anal. Chim. Acta 606, 135 (2008)

2. S. Adams, M. Greeley, Water Air Soil Pollut 123, 103 (2000)

3. M.K. Joshi, P. Mohanty, in Chlorophyll a Fluorescence: A Signature of Photosynthesis, ed. by G.C. Papageorgiou, G.C. Govindjee. (Springer, Dordrecht, 2004), pp. 637-661

4. J.L. Hall, J. Exp. Bot. 53, 1 (2002)

5. R.M. Chaloub, C.C.P. de Magalhaes, C.P. dos Santos, J. Physiol. 41, 1162 (2005)

6. D. Nies, Appl. Microbiol. Biotechnol. 51, 730 (1999)

7. J. Burger, Environ. Bioindic. 1, 136 (2006)

8. C.M. Lu, C.W. Chau, J.H. Zhang, Chemosphere 41, 191 (2000)

9. M.S. Baptista, M.T. Vasconcelos, Crit. Rev. Microbiol. 32, 127 (2006)

10. A.-J. Miao, W.-X. Wang, P. Juneau, Environ. Toxicol. Chem. 24, 2603 (2005)

11. Y. Yu, F. Kong, M. Wang, L. Qian, X. Shi, Ecotoxicol. Environ. Saf. 66, 49 (2007)

12. W.F.J. Vermaas, in Encyclopedia of Life Sciences (Macmillian Reference Ltd., London, 2001), pp. 1-7

13. R.H. Reed, G.M. Gadd, in Heavy Metal Tolerance in Plants: Evolutionary Aspects, ed. by A.J. Shaw. (CRC Press, Boca Raton, FL. 1990), pp. 105-118

14. L.F. de Filippis, C.K. Pallaghy, Z. Pflanzenphysiol. 78, 314 (1992)

15. K. Maxwell, G.N. Johnson, J. Exp. Bot. 51, 659 (2000)

16. N.R. Baker, Annu. Rev. Plant Biol. 59, 89 (2008)

17. N. Mallick, F.H. Mohn, Ecotoxicol. Environ. Saf. 55, 64 (2003)

18. J. Zeng, L. Yang, W.-X. Wang, Aquat. Toxicol. 91, 212 (2009)

19. J. Sikora, J. Żurawski, J. Rutkowska, B. Poniedziałek, K. Wiktorowicz, A. Dudkowiak, Ochr. Środ. Zas. Nat. 41, 293 (2009) 
20. N. Adir, Photosynth. Res. 85, 15 (2005)

21. R. MacColl, Biochim. Biophys. Acta 1657, 73 (2004)

22. W.L. Butler, Annu. Rev. Plant Physiol. 29, 345 (1978)

23. R. Rippka, J. Deruelles, J.B. Waterbury, M. Herdman, R.Y. Stainer, J. Gen. Microbiol. 111, 1 (1979)

24. B. Olejarz, B. Bursa, I. Szyperska, R.-M. Ion, A. Dudkowiak, Int. J. Thermophys. 31, 163 (2010)

25. E. Braslavsky, G.E. Heibel, Chem. Rev. 92, 1381 (1992)

26. T. Gensch, C. Viappiani, Photochem. Photobiol. Sci. 2, 699 (2003)

27. S. Abbruzzetti, C. Viappiani, D.H. Murgida, R. Erra-Balsells, G.M. Bilmes, Chem. Phys. Lett. 304, 167 (1999)

28. A. Planner, D. Fra̧ckowiak, J. Photochem. Photobiol. A 140, 223 (2001)

29. P. Siejak, D. Frąckowiak, J. Photochem. Photobiol. B 88, 126 (2007)

30. R.M.J. Cotterill, Biophysics (Wiley, Chichester, 2002)

31. Y. Pinchasov, T. Berner, Z. Dubinsky, Water Air Soil Pollut. 175, 117 (2006)

32. N. Boucher, R. Carpertier, Photosynth. Res. 59, 167 (1999)

33. S.D.S. Murthy, P. Mohanty, Biol. Plant. 37, 79 (1995) 